\section{BRAF V600E mutation detected in a case of Rosai-Dorfman disease}

Rosai-Dorfman Disease (RDD), also known as sinus histiocytosis with massive lymphadenopathy, is a rare, nonmalignant histiocytic proliferation of unknown etiology. According to the new classification, it belongs to the $\mathrm{R}$ group of histiocytoses. ${ }^{1}$ The clinical course of this disease is quite unpredictable with alternating episodes of worsening and reliving of symptoms. The outcome is usually good, and the disease is often self-limiting. Treatment for RDD is only advised in patients with symptomatic disease or who have a vital organ or system compromised. Common therapeutic modalities include surgery, corticosteroids, interferon, chemotherapy, immunotherapy, and radiotherapy. ${ }^{2}$

Although most individuals with RDD have a benign clinical course, some cases are quite challenging, and optimal target therapeutic agents are missing. The $B R A F^{V 600 E}$ mutation in the proto-oncogene $B R A F$ of the RAS-RAF-MEK-ERK pathway has been consistently reported in histiocytic disorders, including disorders of the non-LCH group, but not in RDD yet. ${ }^{2-5}$

Herein, we report on an 18-year-old man with no previous medical history who presented to his primary care physician with a complaint of progressive bilateral cervical node enlargement in the preceding weeks. Apart from node enlargement, he had no other symptoms (fever, weight loss or night sweats). A node biopsy was obtained, and diagnosis of RDD was established.
The patient was then referred to our center. At the initial evaluation, he maintained nonpainful cervical lymphadenopathy without evidence of airway obstruction or dysphagia (Figure 1A). Physical examination found no neurological signs, skin changes, or hepatosplenomegaly. Laboratory exams were unremarkable, except for an elevated erythrocyte sedimentation rate (ESR) of $25 \mathrm{~mm} / \mathrm{hr}$ (normal range $5-12 \mathrm{~mm} / \mathrm{hr}$ ). A Positron Emission Tomography - Computed Tomography (PET-CT) scan exhibited multiple bilateral hypermetabolic cervical node lesions measuring up to $3 \mathrm{~cm}$ and with maximal standardized uptake value (SUV) of 4.8 (Figure 1B); bone lesions (either hypermetabolic or not) were absent. Slides were reviewed at our center, and a diagnosis of RDD was confirmed.

The diagnosis was independently confirmed by two experts (J.F.E. and F.C.) (Figures 1D and 1E). Immunohistochemical stains demonstrated that large histiocytes were S100 positive (Figure 1F), CD68 positive and CD1a negative. Immunohistochemistry also revealed abundant $B R A F^{V 600 E}$-positive RDD cells (Figure 2A-2B), and disclosed nuclear and cytoplasmic expression of phosphoERK by some typical RDD cells, thus confirming the activation of MAPkinase cell signaling pathway (Figure 2C), Screening for BRAF mutations by pyrosequencing failed to detect a mutation in the p.V600 codon. Nonetheless, the BRAF mutation detected by immunohistochemistry was confirmed by two independent multiplex picodroplet digital PCRs6 (pddPCR) revealed 152 and 124 $B R A F^{V G O O E}$ droplets out of a total of 76,372 and 101,479 droplets analyzed, respectively $(0.16 \%)$ (Figure $2 \mathrm{D})$.
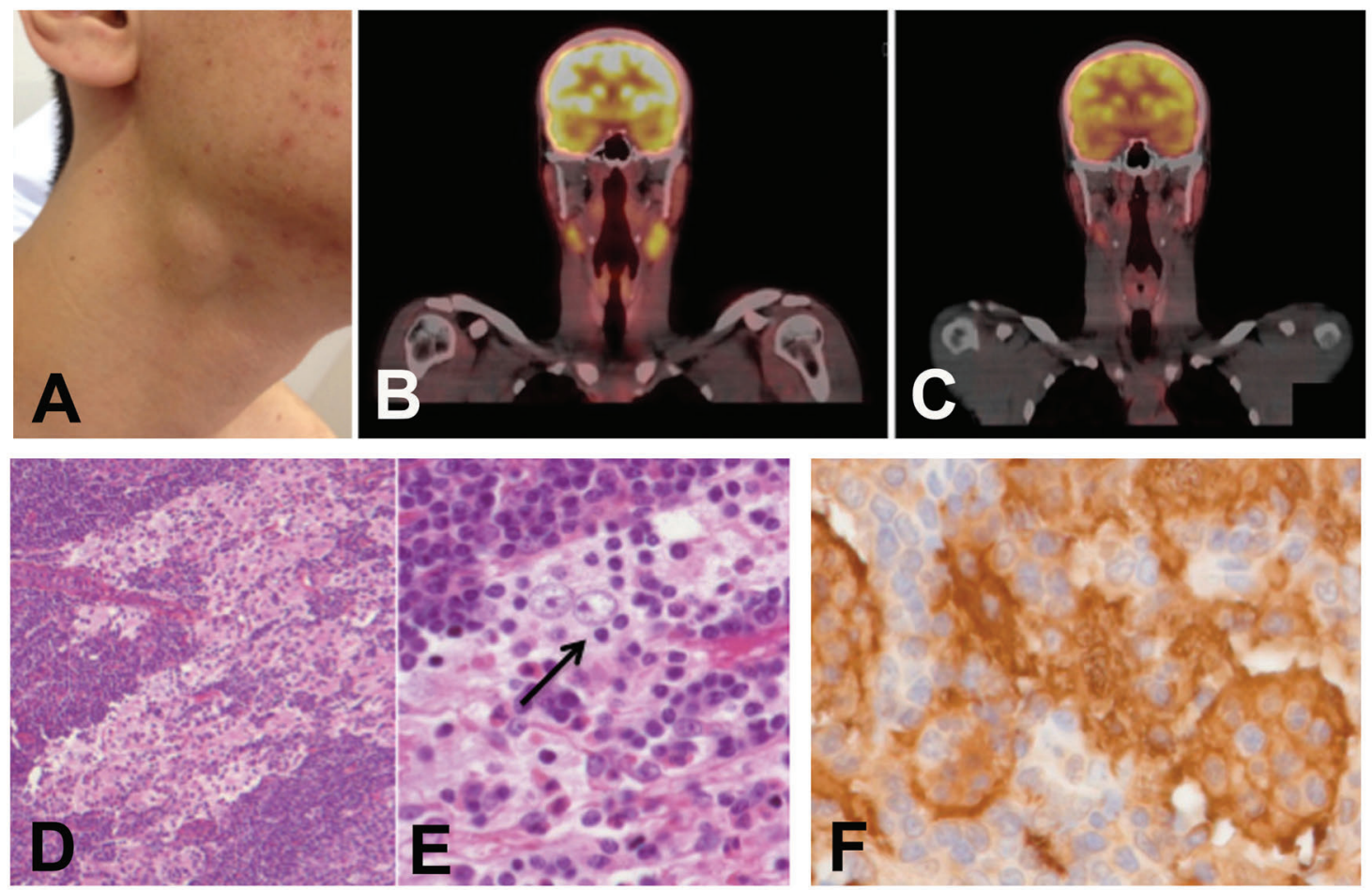

Figure 1. Clinical and histological characteristics of the case. (A) Cervical node enlargement at diagnosis. (B) PET-CT scan at diagnosis showing bilateral hypermetabolic cervical node lesions. (C) Spontaneous decrease in size and metabolism of the previous lesions after eight months of watchful waiting. (D) The lymph node that was biopsied had a preserved architecture, with a massive sinusoidal dilation that contained large plurinucleated histiocytes with large pale nuclei and large nucleoli (H\&E stain, original magnification $\mathrm{x} 40$ ). (E) Emperipolesis lesions were abundant (black arrow), with lymphocytes or plasma cells engulfed within the cytoplasm of histiocytes (H\&E stain, x200). (F) Immunohistochemistry showed strong S100 positivity of histiocytes (x200). 

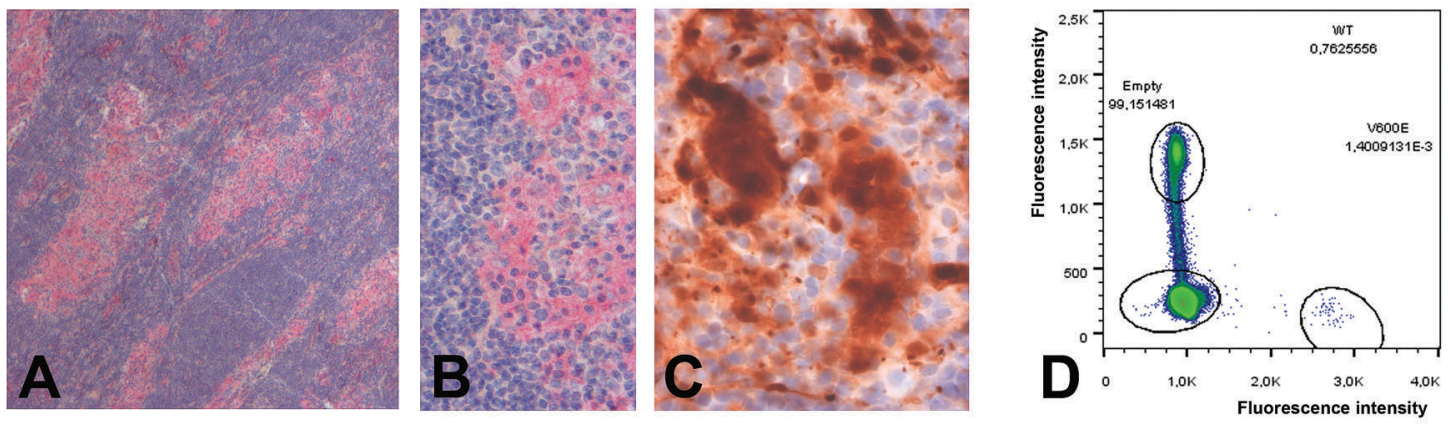

Figure 2. Molecular characteristics of the case. (A-B) Immunohistochemistry with VE1 disclosed abundant typical RDD histiocytes within the sinuses containing the mutant BRAF V600E protein (A x25, B x200). (C) Nuclear and cytoplasmic expression of phosphoERK by typical RDD cells (x200). (D) DNA extracted from areas with the highest density of S100+ histiocytes was analyzed by pddPCR using RainDrop Instrument (RainDance Technologies, Billerica, MA) as described. ${ }^{6}$ Two independent analyzes revealed $0.2 \%$ and $0.12 \%$ of mutant $B R A F^{v 600 E}$.

Since the disease was cervically localized and had been stable, the patient was managed with watchful waiting. Over the next eight months, the cervical nodes showed spontaneous regression. Accordingly, the last follow-up PET-CT scan evidenced marked decrease both in size (up to $1.4 \mathrm{~cm}$ ) and SUV (maximum 3.4) in the previous lesions (Figure 1C).

In the group of histiocytic disorders, the $B R A F^{V 600 E}$ mutation has been found in more than $50 \%$ of cases of $\mathrm{LCH}$ and Erdheim-Chester disease (ECD). ${ }^{1-5}$ Recently, vemurafenib, a BRAF mutant-inhibitor, has shown important clinical and biological response in both $\mathrm{LCH}$ and ECD, corroborating the role of the mutation in the pathogenesis of those disorders. ${ }^{7}$ Concerning the non-L group of histiocytoses, the $B R A F^{V 600 E}$ mutation has only been reported in a few malignant histiocytoses. ${ }^{2-5}$

The distinctive feature about the case we describe is the presence of the $B R A F^{V 600 E}$ mutation. To our knowledge, this is the first report on a BRAF mutation in RDD. In a previous study, we failed to detect any BRAF mutation within 23 different RDD samples. ${ }^{2}$ This result was then confirmed in 13 cases by other authors. ${ }^{3-5}$ The low mutant allelic percentage found in our case may explain the absence of detection in previous studies using other techniques. However, we analyzed 12 other RDD samples with pddPCR with a median of 10,364 droplets analyzed (Range [1,671 to 59,861]), and did not find $B R A F^{V 600 E}$ mutation.

It has been suggested that BRAF mutations could help discriminating between LHC and RDD in the unusual cases in which immunophenotype and morphology findings were equivocal. ${ }^{1}$ However, this case report refutes the usefulness of the $B R A F^{V 600 E}$ mutation for that purpose, even though it might be far more common in LCH compared to RDD. It is of special interest to evaluate if there is a distinct prevalence of the mutation in the systemic form of the disease in comparison to purely cutaneous $\mathrm{RDD}$, which has been pointed out as a separate clinical entity. ${ }^{1,2}$

Regarding treatment implications, we hypothesize vemurafenib, which has been shown to be effective in $\mathrm{LCH}$ and $\mathrm{ECD}, 2,6,7$ might be a promising therapeutic agent in $\mathrm{RDD}$ cases with the $B R A F^{V 600 E}$ mutation. The search for the mutation should be carried out particularly in the refractory and/or recurrent setting. Prospective trials testing vemurafenib in $B R A F^{V 600 E}$-positive RDD and also other histiocytic disorders are needed to further address its role in treating these rare diseases.

The etiology of RDD is largely unknown so far, since a number of presumed causative agents have shown conflicting results. ${ }^{2}$ The polyclonal nature of $\mathrm{RDD}$ has been inferred in early studies. It has been postulated that RDD histiocytes represent macrophage colony-stimulating factor (M-CSF)-induced immune suppressive macrophages with potent T-cell inhibitor effect. According to these findings, $\mathrm{RDD}$ might be an inappropriate, reactive histiocytic proliferation responding to unknown stimuli. Nevertheless, our case shows a clonal $B R A F^{V 600 E}$ mutation, which suggests at least some RDD cases might possess an underlying clonal pathogenesis, and LHC, ECD and some RDD cases may share similar oncogenic pathways, even a common progenitor.

Besides the BRAF ${ }^{V 600}$ mutation, other distinct mutations affecting the MAPK pathway and ultimately causing ERK activation have been described in histiocytoses, ${ }^{8-}$ ${ }^{10}$ and some cases of RDD were reported with upstream RAS or downstream MAP2K1 mutations, also suggesting the clonal nature of this disease. ${ }^{9,11,12}$ Recently successful treatment with MEK inhibitor cobimetinib was reported in one patient. ${ }^{13}$

The pathophysiology of the wide spectrum of clinical manifestations of BRAF-mutated histiocytocytic disorders (varying from indolent to aggressive disease) is still not understood. The state of cell differentiation in which $B R A F^{V 600 E}$ mutation arises seems to determine the clinical extent of the disease; the more primitive the cell harboring the mutation, the more aggressive the disease phenotype. ${ }^{14}$ Loss of oncogene-induced senesce - a protective mechanism characterized by cell-cycle arrest and a robust local inflammatory response - has also been related to clinical aggressiveness in histiocytoses. ${ }^{15}$

Despite these recent promising findings, further studies applying genomic analysis tools (i.e., whole exome/genome sequencing, methylation studies, and RNA sequencing) are warranted in order to better elucidate the pathophysiology of RDD and other histiocytoses and identify eventual therapeutic targets.

Giancarlo Fatobene, Julien Haroche, 2,3 Zofia Hélias-Rodzwicz, ${ }^{4}$ Frédéric Charlotte, ${ }^{2,3}$ Valérie Taly, Aliana Meneses Ferreira, André Néder Ramires Abdo, ${ }^{5,6}$ Vanderson Rocha ${ }^{1,6,7}$ and Jean-François Emile ${ }^{4,8}$

${ }^{1}$ Sírio-Libanês Hospital, São Paulo, Brazil; ${ }^{2}$ AP-HP, Service de Médecine Interne 2, Centre National de Référence des Histiocytoses, 
Hôpital Pitié-Salpêtrière, Paris, France; ${ }^{3}$ Sorbonne Université, Inserm, Centre d'immunologie et des maladies infectieuses-Paris (Cimi-Paris), France; ${ }^{4}$ Department of Pathology, Hôpital Ambroise Paré, AP-HP, Boulogne, France; 'Université Paris Sorbonne Cite, INSERM UMRS775, Paris, France; 'Instituto do Câncer do Estado de São Paulo, Brazil and ${ }^{7}$ Churchill Hospital, Oxford, UK and ${ }^{8}$ EA4340, Versailles University, Boulogne, France

Correspondence: jean-francois.emile@uvsa.fr doi:10.3324/haematol.2018.190934

Information on authorship, contributions, and financial \& other disclosures was provided by the authors and is available with the online version of this article at www. haematologica.org.

\section{References}

1. Emile J-F, Abla O, Fraitag S, et al. Revised classification of histiocytoses and neoplasms of the macrophage-dendritic cell lineages. Blood. 2016;127(22):2672-2681.

2. Haroche J, Cohen-Aubart F, Rollins BJ, et al. Histiocytoses: emerging neoplasia behind inflammation. Lancet Oncol. 2017;18(2):e113-e125.

3. Haroche J, Charlotte F, Arnaud L, et al. High prevalence of BRAF V600E mutations in Erdheim-Chester disease but not in other nonLangerhans cell histiocytoses Brief report High prevalence of BRAF V600E mutations in Erdheim-Chester disease but not in other nonLangerhans cell histiocytoses. Blood. 2012;120(13):2700-2703.

4. Go H, Jeon YK, Huh J, et al. Frequent detection of BRAF V 600E mutations in histiocytic and dendritic cell neoplasms. Histopathology. 2014;65(2):261-272.

5. Chakraborty R, Hampton OA, Shen X, et al. Mutually exclusive recurrent somatic mutations in MAP2K1 and BRAF support a central role for ERK activation in LCH pathogenesis. Blood. 2014; 124(19):3007-3015.

6. Haroche J, Cohen-Aubart F, Emile J-F, et al. Dramatic efficacy of vemurafenib in both multisystemic and refractory Erdheim-Chester disease and Langerhans cell histiocytosis harboring the BRAF V600E mutation. Blood. 2013;121(9):1495-1500.

7. Hyman DM, Puzanov I, Subbiah V, et al. Vemurafenib in multiple nonmelanoma cancers with BRAF V600 mutations. N Engl J Med. 2015;373(8):726-736.

8. Emile J-F, Diamond EL, Helias-Rodzewicz Z, et al. Recurrent RAS and PIK3CA mutations in Erdheim-Chester disease. Blood. 2014; 124(19):3016-3019.

9. Diamond EL, Durham BH, Haroche J, et al. Diverse and targetable kinase alterations drive histiocytic neoplasms. Cancer Discov. 2016;6(2):154-165.

10. Lee LH, Gasilina A, Roychoudhury J, et al. Real-time genomic profiling of histiocytoses identifies early-kinase domain BRAF alterations while improving treatment outcomes. JCI Insight. 2017;2(3):e89473.

11. Garces S, Medeiros LJ, Patel KP, et al. Mutually exclusive recurrent KRAS and MAP2K1 mutations in Rosai-Dorfman disease. Mod Pathol. 2017;30(10):1367-1377.

12. Matter MS, Bihl M, Juskevicius D, Tzankov A. Is Rosai-Dorfman disease a reactve process? Detection of a MAP2K1 L115V mutation in a case of Rosai-Dorfman disease. Virchows Arch. 2017;471(4):545-547.

13. Jacobsen E, Shanmugam V, Jagannathan J. Rosai-Dorfman disease with activating KRAS mutation - response to cobimetinib. N Engl J Med. 2017;377(24):2398-2399.

14. Allen CE, Ladisch S, McClain KL. How I treat Langerhans cell histiocytosis. Blood. 2015;126(1):26-35.

15. Chilosi M, Facchetti F, Caliò A, et al. Oncogene-induced senescence distinguishes indolent from aggressive forms of pulmonary and nonpulmonary Langerhans cell histiocytosis. Leuk Lymphoma. 2014; 55(11):2620-2626. 